\title{
Some operators acting on functional of discrete-time quantum Bernoulli noises
}

\author{
Lixia Zhang, Caishi Wang, Jia Shi, Yin Zhang
}

\begin{abstract}
In this paper, we present some commutation relations of operators, which act on functional of discrete-time quantum Bernoulli noises.
\end{abstract}

Index Terms - quantum Bernoulli noises; com-mut-ation relations; operators

\section{INTRODUCTION}

Quantum Bernoulli noises are the family of annihilation and creation operators acting on square integrable Bernoulli functionals, which can describe a two-level quantum system with infinitely many sites. They satisfy a canonical anti-commutation relation in equal-time and also play an active role in buliding a discrete-time quantum stochastic calculus in infinite dimensions. In 2013, Wang and Zhang considered a kind of localization of quantum Bernoulli noises and showed applications of main results to quantum dynamical semigroups and quantum protum probability. Our work devote to discussing some operators acting on functional of discrete-time quantum Bernoulli noises, which play a key role in Bernoulli functionals. Thus, we consider some commutation relations for the operators, which are interesting.

\section{PRELIMINARIES}

In this section, we briefly recall some notions and results for quantum Bernoulli noises. For details, see [1-2] and references therein.

Let $N$ be the set of all nonnegative integers and $\Gamma$ the finite power set of $N$, namely,

$$
\Gamma=\{\sigma \mid \sigma \subset N \text { and } \# \sigma<\infty\},
$$

where $\# \sigma$ denotes the cardinality of $\sigma$ as a set.

Thoughout, we assume that $(\Omega, F, P)$ is a probability space and $Z=\left(Z_{n}\right)_{n \geq 0}$ is an independent sequence of random variables on $(\Omega, F, P)$, which satisfies that

$P\left\{Z=\theta_{n}\right\}=p_{n}, \quad P\left\{Z=-1 / \theta_{n}\right\}=q_{n}, \quad n \geq 0$ with $\theta_{n}=\sqrt{q_{n} / p_{n}}, q_{n}=1-p_{n}$ and $0 \leq p_{n} \leq 1$. And, moreover, $F=\sigma\left(Z_{n}, n \geq 0\right)$, the $\sigma$-filed generated by $Z=\left(Z_{n}\right)_{n \geq 0}$. And $Z$ is actually a discrete-time Bernoulli noise.

Lixia Zhang, School of Mathematics and Statistics, Northwest Normal University, Lanzhou, China, Mobile, 86-15193197021.

Caishi Wang, School of Mathematics and Statistics, Northwest Normal University, Lanzhou, China.

Yin Zhang, School of Mathematics and Statistics, Northwest Normal University, Lanzhou, China.

Jia Shi, School of Mathematics and Statistics, Northwest Normal University, Lanzhou, China.
Let $L^{2}(Z)$ be the space of square integrable complex-valued random variables on $(\Omega, F, P)$.

We denote by $\langle\cdot, \cdot\rangle$ the inner product of $L^{2}(Z)$, and by $\|, \cdot\|$ the corresponding norm. It is known that $Z$ has the orthonormal basis $\left\{Z_{\sigma} \mid \sigma \in \Gamma\right\}$, where $Z_{\varnothing}=1$ and

$$
Z_{\sigma}=\prod_{i \in \sigma} Z_{i}, \sigma \in \Gamma, \sigma \neq \varnothing \text {, }
$$

which shows that $L^{2}(Z)$ is an infinite dimensional space.

For $k \geq 0$, we use $E_{k}$ to denote the conditional exceptation given $\sigma$-filed $F_{k}$, namely

$$
E_{k}=E\left[\cdot \mid F_{k}\right]
$$

where $F_{k}$ is the $\sigma$-filed generated by $\left(Z_{j}\right)_{0 \leq j \leq k}$. It is known that $E_{k}$ is a projection operator on $L^{2}(Z)$ and its range is $L^{2}\left(\Omega, F_{k}, P\right)$, which is a $2^{k+1}$-dimensional of $L^{2}(Z)$.

Lemma 1.[1] For $k \geq 0$, there exists a bounded operator $\partial_{k}$ on $L^{2}(Z)$ such that

$\partial_{k} Z_{\sigma}=1_{\sigma}(k) Z_{\sigma \backslash k,} \quad \sigma \in \Gamma$, where $\sigma \backslash k=\sigma \backslash\{k\}$ and $1_{\sigma}(k)$ is the indicator of $\sigma$ a subset of $N$.

Lemma 2.[1] For $k \geq 0$, then $\partial_{k}^{*}$, the adjoint operator, has following property:

$$
\partial_{k}^{*} Z_{\sigma}=\left(1-1_{\sigma}(k)\right) Z_{\sigma \cup k,} \quad \sigma \in \Gamma,
$$

where $\sigma \cup k=\sigma \cup\{k\}$.

Lemma 3. [2] For $k \geq 0$, we call $l_{k}=\partial_{k} E_{k}$ the local annihilation and its adjoint operator $l_{k}^{*}=\partial_{k}^{*} E_{k}$ the local creation operator.

Lemma 4.[2] For $k \geq 0$, we have

$$
l_{k}^{*} l_{k}=\left(\partial_{k}^{*} \partial_{k}\right) E_{k} \text {. }
$$




\section{MAIN RESULTS}

Definition 1. A diagonal operator $C$ on functionals of $Z$ is defined as

$$
C x=\sum_{\sigma \in \Gamma} \lambda_{\sigma}^{p}\left\langle Z_{\sigma}, x\right\rangle Z_{\sigma}, x \in \operatorname{Dom} C, p \geq 0,
$$

with

$$
\text { Dom } C=\left\{\left.x \in L^{2}(z)\left|\sum_{\sigma \in \Gamma} \lambda_{\sigma}^{2 p}\right|\left\langle Z_{\sigma}, x\right\rangle\right|^{2}<\infty\right\},
$$

where

$$
\lambda_{\sigma}= \begin{cases}\prod_{\sigma \in \Gamma}(k+1), & \sigma \neq \varnothing, \sigma \in \Gamma ; \\ 1, & , \sigma=\varnothing, \sigma \in \Gamma .\end{cases}
$$

Clearly, Dom C contains the canonical orthonormal basis of $\left\{Z_{\sigma} \mid \sigma \in \Gamma\right\}$, which means the $C$ is a densely defined operator in $L^{2}(Z) . L^{2}(Z)$ has an orthonormal basis of $\left\{Z_{\sigma} \mid \sigma \in \Gamma\right\}$. Thus, for each $n \geq 0$, we put

$$
\begin{aligned}
H_{n} & =\operatorname{span}\left\{Z_{\sigma} \mid \sigma \in \Gamma, \sigma \subset[0, n]\right\} \\
& =\operatorname{span}\left\{Z_{\sigma} \mid \sigma \in \Gamma_{n]}\right\} .
\end{aligned}
$$

Clearly, for each $n \geq 0, H_{n} \subset L^{2}(Z)$ and the dimension of $H_{n}$ is $2^{n+1}$, which means that $H_{n}$ is a closed subspace of $L^{2}(Z)$ and $\left\{Z_{\sigma} \mid \sigma \in \Gamma_{n]}\right\}$ is an orthonormal basis of $H_{n}$.

Definition 2. For $n \geq 0, P_{n}$ is the projection operator from $L^{2}(Z)$ onto $H_{n}$, namely, for all $x \in L^{2}(Z), P_{n} x=\sum_{\sigma \in \Gamma_{n]}}\left\langle Z_{\sigma}, x\right\rangle Z_{\sigma}$.

Theorem 1. Let $p \geq 0$ be a nonnegative real number. Then, for all $n \geq 0, C P_{n}$ makes sense, and moreover $C P_{n}=P_{n} C$ on Dom $C$.

Proof. Let $n \geq 0$. Then, $H_{n} \subset L^{2}(Z) \subset \operatorname{Dom} C$, which together with the fact that $H_{n}$ is just the range of $P_{n}$, impiles that $C P_{n}$ makes sense. Now, for $x \in \operatorname{Dom} C$, it follows from the definitions of $C$ and $P_{n}$ that

$$
\begin{gathered}
C P_{n} x=\sum_{\sigma \in \Gamma} \lambda_{\sigma}^{p}\left\langle Z_{\sigma}, P_{n} x\right\rangle Z_{\sigma}=\sum_{\sigma \in \Gamma} \lambda_{\sigma}^{p}\left\langle P_{n} Z_{\sigma}, x\right\rangle Z_{\sigma} \\
=\sum_{\sigma \in \Gamma_{n]}} \lambda_{\sigma}^{p}\left\langle Z_{\sigma}, x\right\rangle Z_{\sigma},
\end{gathered}
$$

and

$$
\begin{aligned}
P_{n} C x=P_{n} & \sum_{\sigma \in \Gamma} \lambda_{\sigma}^{p}\left\langle Z_{\sigma}, x\right\rangle Z_{\sigma}=\sum_{\sigma \in \Gamma} \lambda_{\sigma}^{p}\left\langle Z_{\sigma}, x\right\rangle P_{n} Z_{\sigma} \\
& =\sum_{\sigma \in \Gamma_{n]}} \lambda_{\sigma}^{p}\left\langle Z_{\sigma}, x\right\rangle Z_{\sigma},
\end{aligned}
$$

which givens $C P_{n} x=P_{n} C x$.

Theorem 2. Let $p \geq 0$ be a nonnegative real number. Then, for all $k \geq 0$, both $l_{k}^{*} l_{k} C$ and $C l_{k}^{*} l_{k}$ make sense on $L^{2}(Z)$, and moreover it holds on $L^{2}(Z)$ that $C l_{k}^{*} l_{k}=l_{k}^{*} l_{k} C$.

Proof. Let $k \geq 0$. It is easy to see that $\operatorname{Dom}_{k}^{*} l_{k}=L^{2}(Z)$, which together with the fact $L^{2}(Z) \subset \operatorname{Dom} C$, implies that $C l_{k}^{*} l_{k}$ makes sense on $L^{2}(Z)$. Similarly, $l_{k}^{*} l_{k} C$ also makes sense on $L^{2}(Z)$. To complete the proof, it suffices to show that $C l_{k}^{*} l_{k} Z_{\sigma}=l_{k}^{*} l_{k} C Z_{\sigma}$ holds for all $\sigma \in \Gamma$, in fact, for all $\sigma \in \Gamma$, by (1), (2) and definition of $C$, we have

$$
\begin{gathered}
C l_{k}^{*} l_{k} Z_{\sigma}=C\left[\left(\partial_{k}^{*} \partial_{k}\right) E_{k} Z_{\sigma}\right]=C 1_{\max \sigma=k} Z_{\sigma} \\
=1_{\max \sigma=k} C Z_{\sigma}=1_{\max \sigma=k} \sum_{\sigma \in \Gamma} \lambda_{\sigma}^{p}\left\langle Z_{\sigma}, Z_{\sigma}\right\rangle Z_{\sigma} \\
=1_{\max \sigma=k} \sum_{\sigma \in \Gamma} \lambda_{\sigma}^{p} Z_{\sigma},
\end{gathered}
$$

and

$$
\begin{aligned}
l_{k}^{*} l_{k} C Z_{\sigma} & =l_{k}^{*} l_{k} \sum_{\sigma \in \Gamma} \lambda_{\sigma}^{p}\left\langle Z_{\sigma}, Z_{\sigma}\right\rangle Z_{\sigma} \\
& =l_{k}^{*} l_{k} \sum_{\sigma \in \Gamma} \lambda_{\sigma}^{p} Z_{\sigma}=\sum_{\sigma \in \Gamma} \lambda_{\sigma}^{p} l_{k}^{*} l_{k} Z_{\sigma} \\
& =\sum_{\sigma \in \Gamma} \lambda_{\sigma}^{p}\left(\partial_{k}^{*} \partial_{k}\right) E_{k} Z_{\sigma}=1_{\max \sigma=k} \sum_{\sigma \in \Gamma} \lambda_{\sigma}^{p} Z_{\sigma} .
\end{aligned}
$$

which givens $C l_{k}^{*} l_{k} Z_{\sigma}=l_{k}^{*} l_{k} C Z_{\sigma}$.

Theorem 3. For all $k, n \geq 0, l_{k}^{*} l_{k} P_{n}$ makes sense on $L^{2}(Z)$, and moreover it holds on $L^{2}(Z)$ that $P_{n} l_{k}^{*} l_{k}=l_{k}^{*} l_{k} P_{n}$.

Proof. Let $k \geq 0$. It is easy to see that $\operatorname{Dom}_{k}^{*} l_{k}=L^{2}(Z)$. Obviously, $l_{k}^{*} l_{k} P_{n}$ makes sense. Now, we prove $P_{n} l_{k}^{*} l_{k}=l_{k}^{*} l_{k} P_{n}$. In fact, it suffices to show that $P_{n} l_{k}^{*} l_{k} Z_{\sigma}=l_{k}^{*} l_{k} P_{n} Z_{\sigma}$ holds for all $\sigma \in \Gamma$, by (1), (2) and definition of $P_{n}$, we have

$$
\begin{aligned}
P_{n} l_{k}^{*} l_{k} Z_{\sigma} & =P_{n}\left[\left(\partial_{k}^{*} \partial_{k}\right) E_{k} Z_{\sigma}\right] \\
& =P_{n} 1_{\max \sigma=k} Z_{\sigma}=1_{\max \sigma=k} P_{n} Z_{\sigma}, \sigma \in \Gamma_{n]},
\end{aligned}
$$

and

$l_{k}^{*} l_{k} P_{n} Z_{\sigma}=l_{k}^{*} l_{k} Z_{\sigma}=\left(\partial_{k}^{*} \partial_{k}\right) E_{k} Z_{\sigma}, \sigma \in \Gamma_{n]}$

which givens $P_{n} l_{k}^{*} l_{k} Z_{\sigma}=l_{k}^{*} l_{k} P_{n} Z_{\sigma}$.

\section{ATCKNOWLEDGEMENT}

This work is supported by National Natural Science Foundation of China (Grant No. 11461061).

\section{REFERENCE}

[1] Wang C.S, Chai H.F, Lu Y.C. Discrete-time quantum Bernoulli noises[J]. Journal of Mathematical Physics,51(5): 8(2010)

[2] Wang C.S, Zhang J.H. Localization of quantum Bernoulli noises[J]. Journal of Mathematical Physics,54(10): 103502-103509(2013).

[3] N. Privault, Stochastic analysis of Bernoulli processes, Probab. Surv. 5 (2008), 435-483.

[4 ]C.S. Wang, Y.C. Lu and H.F. Chai, An alternative approach to Privault's discrete-time chaotic calculus, J. Math. Anal. Appl. 373(2011), 643--654

[5]C.S. Wang and J.H. Zhang, Wick analysis for Bernoulli noise functionals, Journal of Function Spaces, Volume 2014, Article ID 727341.

Lixia Zhang, School of Mathematics and Statistics, Northwest Normal University, Lanzhou, China, Mobile, 86-15193197021.

Caishi Wang, School of Mathematics and

Statistics, Northwest Normal University, Lanzhou, China.

Yin Zhang, School of Mathematics and Statistics, Northwest Normal University, Lanzhou, China.

Jia Shi, School of Mathematics and Statistics, Northwest Normal University, Lanzhou, China. 